Conclusions The present study shows that the risk of cancer is highly dependent on occupation and specific occupational exposures.

\section{D.3 MEDICATION AS PROXY OF WORK-RELATED HEALTH PROBLEMS}

${ }^{1}$ Lode Godderis*, ${ }^{2}$ Lieve Vandersmissen, ${ }^{2}$ Martijn Schouteden. ${ }^{1} K U$ Leuven/idewe, Leuven, Belgium; ${ }^{2}$ IDEWE, Heverlee, Belgium

\subsection{6/OEM-2019-EPI.74}

Introduction In order to investigate the impact of work on health, we hypothesize that medication consumption registered in medical files of workers could serve as a proxy for workrelated health problems. In this study, we describe variations in prevalence of specific medication groups between sectors, adjusting for age and gender. In addition, we investigated whether a change in job/sector can have an impact on medication use.

Method Logistic regression analysis is being performed to investigate the effect of occupational sector on the prevalence of specific medication groups, adjusted for year, age and gender. For this, an occupational surveillance dataset of 686434 workers collected between 2011 and 2017 was used. Additionally, regarding the impact on job changes on medication use, analyses are currently being performed by comparing prevalence of specific medication groups in 2011 with 2017 for those employees who changed job during this time period.

Results and discussion In 2011 30,6\% male and 49,8\% female workers used medication. These figures roze to $43,1 \%$ and $67,3 \%$ respectively in 2017 . The use of medication increased with age: in $201738,2 \%$ for workers $<25$ year, $43,6 \%$ for 25-34 year old employees, 48,7\% between $35-44$ year, $61,6 \%$ between 45-54 year and 74,1\% for older workers $>=55$ year. Big differences were observed between sectors. Medication use was highest in health care $(67,1 \%$ in 2017$)$, government and education. These differences remained after adjustment for age and gender. $9,8 \%$ and $9,1 \%$ of the workers were treated for respectively pain and neuropsychological disorders in 2017.

Conclusion Significant differences in workers' medical consumption were observed between sectors. This information is now being used for the implementation of a sector-oriented health surveillance program.

\section{D.4 INNOVATIVE APPROACHES TO DATA ACQUISITION, STANDARDIZATION, POOLING AND ANALYSES OF OCCUPATIONAL HEALTH INFORMATION}

Roel Vermeulen*. Utrecht University, Utrecht, Netherlands

\subsection{6/OEM-2019-EPI.75}

Occupation and employment is a major determinant of health and healthy aging. Despite the temporal increase in time spent in employment and profound changes in working life, there is limited coordinated research on occupation and health.

We recently estimated that in Europe alone there is information available on more than 30 million individuals that could be used to study the association between working life and health. However, lack of standardization, inability to code large numbers of job-entries and inabilities to share data have hindered progress. In order to unlock the vault of occupational-health information novel ways of data acquisition, standardization, pooling and analyses have to be developed and implemented. The ability of pooling studies would greatly improve occupational health studies by increasing sample size for robust inference, and would allow risk stratification, identification of new risks, exploration of interactions with work and non-work-related factors and detailed analyses on the shapes of the exposure-response curves.

In recent years we have seen progress in several different methodological aspects. Currently, new tools are available to collect information on work and work exposures. These include amongst others the use of sensors and mobile phone applications. Standardization of occupational information is essential for comparison of results between studies and for pooling of studies. This could be achieved by using common occupational coding schemes, use of automatic coding instruments and common exposure assessment tools. Virtual pooling of data has become possible by using data-shields which instead of bringing the data to the analyses bring the analyses to the data allowing federated analyses.

As part of the OMEGA-NET symposium we will discuss the need for new tools and avenues of how to bring occupational health information together. We will present our inventory of novel tools to aid this process, and will discuss future needs.

\section{O3D.5 NATIONAL POLICIES AND SOCIAL INEQUALITIES IN EXIT PATHS FROM WORKING LIFE IN SWEDEN}

1,2 Kerstin Nilsson, ${ }^{3}$ Roland Kadefors, ${ }^{4}$ Per-Olof Östergren, 'Lars Rylander, ${ }^{1,5}$ Maria Albin*.
${ }^{1}$ Division of Occupational and Environmental Medicine, Lund University, Lund, Sweden;
${ }^{2}$ Department of Work Science, Swedish University of Agricultural Sciences, Alnarp, Sweden;
${ }^{3}$ Department of Sociology and Work Science, University of Gothenburg, Gothenburg,
Sweden; ${ }^{4}$ Division of Social Medicine and Global Health, Lund University, Lund, Sweden;
${ }^{5}$ Unit of Occupational Medicine, Institute of Environmental Medicine, Karolinska Institutet,
Stockholm, Sweden

10.1136/OEM-2019-EPI.76

We investigated the impact on work life exits from reduced access to disability pension (2006), and financial incentives to extend working life.

Method and material We used labour statistics, social insurance, and income data, for all employees in Sweden, to compare occupational groups (SSYK, based on ISCO-88), and blue and white collar workers, with regard to i) lost years in working life due to death, disability pension and long-term sickleave preceding disability pension 2007-2010, ii) granted disability pensions 2007-2011, and iii) premature age pension in 2004 and 2011.

Results Years lost in working life were similar for men and women in the same 1-digit SSYK occupational group, somewhat higher for those born outside Sweden, but showed a clear gradient from white to blue collar occupations, e.g. on average 0.39 ys versus 2.40 ys lost for Legislators/senior officials/managers and in Elementary occupations, respectively (women born in Sweden).

In 2006 the prevalence of disability pension in the age group 50-64 was $3.61 \%$ among women and $2.49 \%$ among men, with $10 / 10$ of the highest prevalence occupations (4-digit SSYK code) in men, and $9 / 10$ in women, being blue collar ones. Approved applications decreased $2006-2011$ by $74.4 \%$ in women, and $64.3 \%$ in men; for mental disorders (ICD-10- 